\title{
Fermi-edge singularity in nonequilibrium systems
}

\author{
Tai-Kai Ng \\ Department of Physics, Hong Kong University of Science and Technology, Clear Water Bay Road, Kowloon, Hong Kong
}

(Received 28 June 1995)

\begin{abstract}
In this paper the problem of Fermi-edge singularity is studied in detail for a class of nonequilibrium systems with more than one Fermi seas with different chemical potentials coupling to each other. The problem is solved by generalizing the solution of Noziéres and De Domincis for (equilibrium) single Fermi sea system to the nonequilibrium situation described in this paper. The concepts of nonequilibrium $T$ matrices and phase shifts are introduced in this paper where a generalized Fumi theorem is being proved. We find that Fermi-edge singularities are found in all Fermi surfaces in the system. However the singularities are smeared out by a lifetime effect associated with finite potential differences between different Fermi seas. Some consequences of the result are pointed out. [S0163-1829(96)10431-8]
\end{abstract}

\section{INTRODUCTION}

Recently there are much interests in the study of nonlinear ${ }^{1-8}$ and time-dependent ${ }^{9,10}$ transports in mesoscopic systems where interesting physics associated with quantum coherence in nonequilibrium situations are expected to be uncovered. For example, $\mathrm{Ng}^{1}$ has proposed the possibility of observing different $I-V$ characteristics in voltage-driven and current-driven experiments as a result of thermodynamics. Quantum coherence effects associated with electronic interaction in a nonequilibrium environment was also proposed in shot-noise measurement ${ }^{6,7}$ and in resonant tunneling systems. $^{2-5}$ More generally, one may ask the question of where one should look for new quantum mechanical phenomena for mesoscopic systems in a nonequilibrium state? To answer the question (for steady state transports), we notice that the typical voltage difference applied on the system in a mesoscopic experiment is of order a few $\mathrm{meV}$ (or less), which is much less than typical microscopic energy scales in the system. The small voltage applied on the system implies that the underlying properties of the sample will not be altered too much away from its equilibrium behaviors. Only those properties which are very sensitive to the structure of Fermi surfaces will be significantly affected. In this paper we shall consider one class of phenomena which have precisely this property - the problem of Fermi-edge singularity. ${ }^{11-13}$ We shall examine how the phenomenon got modified in the presence of more than one Fermi surface with different chemical potentials and shall point out several applications of this problem to out of equilibrium mesoscopic phenomena. A Brief Report of our results has been published elsewhere. ${ }^{14}$ In this paper, details of our study will be presented. In particular the concept of nonequilibrium $T$ matrices and phase shifts will be introduced rigorously where a generalized Fumi theorem will be proved. An explicit example with two Fermi seas will be worked out in detail. The importance of eigenvalues and eignvectors of the (nonequilibrium) $T$ matrices in solving the Fermi-edge singularity problem will be pointed out.

The problem we study can be summarized by the following time-dependent one-particle Hamiltonian, ${ }^{12,13}$ $H=H_{o}+V(t)$, where,

$$
H_{o}=\sum_{\eta, \epsilon_{k}<\mu_{\eta}} \epsilon_{k} c_{k \eta}^{\dagger} c_{k \eta}
$$

and

$$
V(t)=\sum_{k \eta, k^{\prime} \eta^{\prime}} V_{\eta, \eta^{\prime}} c_{k \eta^{\dagger}}^{\dagger} c_{k^{\prime} \eta^{\prime}} \theta\left(t_{f}-t\right) \theta\left(t-t_{i}\right)
$$

where $\eta, \eta^{\prime}=1, \ldots, N$ are Fermi sea indices and $\mu_{\eta}$ is the chemical potential for Fermi sea $\eta$. Notice that we have neglected spin in our analysis. In the usual Fermi-edge problem, $N=1$ (i.e., only one Fermi surface present) and $t_{i}$ and $t_{f}$ are the beginning and ending times of an x-ray emission (absorption) process. In this case, the problem was solved by Noziéres and De Domincis (ND) (Ref. 12) who showed that the $\mathrm{x}$-ray emission (absorption) spectrum can be obtained from the one-particle Green's function $F_{k, k^{\prime}}\left(t, t^{\prime}\right)$ $=\left\langle T c_{k}(t) c_{k^{\prime}}^{\dagger}\left(t^{\prime}\right)\right\rangle$ of the system in the presence of the timedependent potential $V(t)$. The new feature in the nonequilibrium problem is that the time-dependent potential $V(t)$ now involves scattering of electrons between different Fermi seas where the chemical potentials are not equal. Notice that "sharp" Fermi surfaces are still present in our model implying that the Fermi-edge singularity is not removed automatically in the nonequilibrium problem. Notice also that eigenstates of $H_{o}$ characterized by electron operators $c_{k \eta}$ 's are, in general scattering states with electron waves incoming from Fermi sea $\eta$ with wave vector $k .{ }^{1} \mathrm{~A}$ related non-equilibrium Fermi-edge problem with only one Fermi sea has also been studied by Tanguy and Combescot. $^{15,16}$ The multi-Fermi seas problem is qualitatively very different from the single Fermi-sea problem when the system is out of equilibrium as we shall see later.

As in the usual Fermi-edge singularity problem, we shall employ the " $s$-wave scattering only" approximation ${ }^{12}$ and consider the one-particle Green's functions $F_{\eta \eta^{\prime}}\left(t, t^{\prime}\right)=\Sigma_{k, k^{\prime}}\left\langle T c_{k \eta}(t) c_{k^{\prime} \eta^{\prime}}^{\dagger}\left(t^{\prime}\right)\right\rangle$ in the presence of the external potential $V(t)$. In the interaction representation,

$$
F_{\eta \eta^{\prime}}\left(t, t^{\prime}\right)=\sum_{k, k^{\prime}}\left\langle\psi_{o}\left|T c_{k \eta}(t) c_{k^{\prime} \eta^{\prime}}^{\dagger}\left(t^{\prime}\right) S\left(t_{f}, t_{i}\right)\right| \psi_{o}\right\rangle
$$


where $S\left(t_{f}, t_{i}\right)=T\left(\exp -i / \hbar \int_{t_{i}}^{t_{f}} V\left(t^{\prime}\right) d t^{\prime}\right)$ is the time evolution operator under $V\left(t^{\prime}\right)$ and $\left|\psi_{o}\right\rangle$ is the wave function of the system before $V(t)$ is switched on. In particular, we are interested at the limit $t \rightarrow t_{f}$ and $t^{\prime} \rightarrow t_{i}$ where the physical photoemission (absorption) spectrum can be obtained from $F_{\eta \eta^{\prime}}\left(t_{f}, t_{i}\right) .{ }^{12}$ In our nonequilibrium problem, $\left|\psi_{o}\right\rangle$ describes a state with $N$ Fermi seas filled up to their respective chemical potentials $\mu_{\eta}$ and the difference between equilibrium and nonequilibrium systems is reflected in difference choices of $\left|\psi_{o}\right\rangle$ only. This is very different from the usual treatment of electron interaction in nonequilibrium systems where the one-electron Green's function of interest is

$$
D\left(k t, k^{\prime} t^{\prime}\right)=\left\langle\psi_{o}\left|S(-\infty, \infty) T c_{k}(t) c_{k^{\prime}}^{\dagger}\left(t^{\prime}\right) S(\infty,-\infty)\right| \psi_{o}\right\rangle,
$$

with the time-evolution operator goes through a closed time loop from $-\infty$ to $\infty$ and then back to $-\infty$. In particular, the nonequilibrium Fermi-edge problem can be treated by the standard time-ordered Green's function perturbation technique whereas the Keldysh technique is required in treating problems where close-time-loop evolution is present.

Following standard diagramatic analysis, ${ }^{11}$ it can be shown that the Green's function $F$ can be expressed as the product of two terms,

$$
F_{\eta \eta^{\prime}}\left(t, t^{\prime}\right)=G_{\eta \eta^{\prime}}\left(t, t^{\prime}\right) e^{C\left(t_{f}, t_{i}\right)},
$$

where $G_{\eta \eta^{\prime}}\left(t, t^{\prime}\right)$ includes the sum of all connected diagrams and $C\left(t_{f}, t_{i}\right)$ includes the sum of all single closed-loop diagrams by the Linked Cluster theorem. ${ }^{11}$ The connected Green's functions $G_{\eta \eta^{\prime}}$ satisfy the Dyson equation ${ }^{12}$

$$
\begin{aligned}
G_{\eta \eta^{\prime}}\left(t, t^{\prime}\right)= & \delta_{\eta \eta^{\prime}} g_{\eta}\left(t-t^{\prime}\right)+i \sum_{\eta^{\prime \prime}} V_{\eta \eta^{\prime \prime}} \\
& \times \int_{t_{i}}^{t_{f}} g_{\eta}(t-t \prime \prime) G_{\eta^{\prime \prime} \eta^{\prime}}\left(t^{\prime \prime}, t^{\prime}\right) d t^{\prime \prime}
\end{aligned}
$$

where $g_{\eta}\left(t-t^{\prime}\right)$ is the unperturbed Green's function governed by $H_{o}$. The closed-loop contribution $C\left(t_{f}, t_{i}\right)$ can be obtained from connected Green's functions $G_{\eta \eta^{\prime}}(t, t)$ 's following a trick of coupling constant integration introduced by $\mathrm{ND},{ }^{12}$

$$
\left(\lambda \frac{\partial C\left(t_{f}-t_{i}\right)}{\partial \lambda}\right)=i \sum_{\eta, \eta^{\prime}}\left(\lambda V_{\eta \eta^{\prime}}\right) \int_{t_{i}}^{t_{f}} G_{\eta^{\prime} \eta}^{\lambda}(t, t) d t
$$

where $G_{\eta \eta^{\prime}}^{\lambda}\left(t, t^{\prime}\right)$ 's are connected Green's functions satisfying the Dyson equation (3) with $V_{\eta \eta^{\prime}} \rightarrow \lambda V_{\eta \eta^{\prime}}$. Equations (3) and (4) are direct consequences of formal perturbation theory and are valid independent of precise form of the initial state $\left|\psi_{o}\right\rangle$.

To solve the Dyson equation (3), we have to know the unperturbed Green's function $g_{\eta}$ 's. The exact form of $g_{\eta}$ depends on the detail behavior of $H_{o}$ and cannot be obtained analytically in general. Following $\mathrm{ND},{ }^{12}$ we consider the approximate expression

$$
\begin{aligned}
g_{\eta}\left(t-t^{\prime}\right)= & -i \nu_{o \eta}\left(P \frac{1}{\left(t-t^{\prime}\right)} e^{-i \mu_{\eta}\left(t-t^{\prime}\right)}\right. \\
& \left.+\pi \tan \theta_{o \eta} \delta\left(t-t^{\prime}\right)\right)
\end{aligned}
$$

where $\nu_{o \eta}$ is the density of states for Fermi sea $\eta$. The first term in Eq. (5) gives the correct long-time asymptotic behavior of $g_{\eta}(t)$ arising from the Fermi surface discontinuity, whereas the second term describes approximately the shorttime behavior of $g_{\eta}{ }^{12}$ Notice that in this approximation, difference in the positions of Fermi surfaces for different Fermi seas is reflected only in the phase factor $e^{-i \mu_{\eta}\left(t-t^{\prime}\right)}$. Correspondingly, in solving Eq. (3), the effect of more than one chemical potential shows up only as an interference effect between different Green's functions $g_{\eta}$ 's.

In the following sections we shall study the long-time asymptotic solution of the Dyson equation and the corresponding Fermi-edge singularity. In Sec II we shall first consider the infinite time $\left(t_{f} \rightarrow \infty, t_{i} \rightarrow-\infty\right)$ problem where the Dyson equation can be solved easily. We shall discuss in detail the mathamatical structure of the solution where appearance of complex phase shifts in the problem will be pointed out. The physical reason for the appearance of this nonunitarity behavior will be explained. In Sec. III we shall discuss the solution of the Dyson equation for finite $t_{f}, t_{i}$ in detail. The long-time behvior of the Green's functions $G_{\eta \eta^{\prime}}\left(t_{f}, t_{i}\right)$ and $C\left(t_{f}, t_{i}\right)$ will be computed. The results will be applied to study the photoemission (luminescence) spectrum of a two Fermi-sea system in Sec. IV where we shall also summarize and discuss some other plausible applications of our result.

\section{SOLUTION OF DYSON EQUATION IN INFINITE TIME LIMIT}

The Dyson equation with the approximate form of $g_{\eta}(t)$ can be solved easily in the limit $t_{i} \rightarrow-\infty, t_{f} \rightarrow \infty$ by Fourier transforming Eq. (3). The solution can be represented conveniently by introducing matrices $\mathbf{G}(\omega)$ and $\mathbf{g}(\omega)$, where $[\mathbf{G}(\omega)]_{\eta \eta^{\prime}}=G_{\eta \eta^{\prime}}(\omega)$ and $[\mathbf{g}(\omega)]_{\eta \eta^{\prime}}=\delta_{\eta \eta^{\prime}} g_{\eta}(\omega)$, where $G_{\eta \eta^{\prime}}(\omega)$ and $g_{\eta}(\omega)$ are Fourier transforms of $G_{\eta \eta^{\prime}}\left(t-t^{\prime}\right)$ and $g_{\eta}\left(t-t^{\prime}\right)$, respectively. In matrix notation, the Dyson Equation becomes

$$
\mathbf{G}(\omega)=\mathbf{g}(\omega)+i \mathbf{g}(\omega) \mathbf{V G}(\omega)
$$

where $[\mathbf{V}]_{\eta \eta^{\prime}}=V_{\eta \eta^{\prime}}$ is the interaction matrix. Fourier transforming Eq. (5), we obtain also

$$
g_{\eta}(\omega)=\nu_{o \eta} \pi\left[-i \tan \theta_{\eta}+\operatorname{sgn}\left(\omega-\mu_{\eta}\right)\right] .
$$

Notice that the matrix $\mathbf{g}(\omega)$ is independent of frequency $\omega$ except when crossing chemical potentials $\omega=\mu_{\eta}$ where the real part of $\mathbf{g}(\omega)$ changes discontinously. Correspondingly, we expect that the solution of Eq. (6a) $\left\{\mathbf{G}(\omega)=[\mathbf{I}-i \mathbf{g}(\omega) \mathbf{V}]^{-1} \mathbf{g}(\omega)\right\}$ should also have similar behavior, i.e., we expect

$$
\mathbf{G}(\omega)=\sum_{i=1}^{N} \mathbf{G}_{d}{ }^{i} \operatorname{sgn}\left(\omega-\mu_{i}\right)+\mathbf{G}_{c},
$$

where $\mathbf{G}_{d}{ }^{i}$ and $\mathbf{G}_{c}$ are $N \times N$ frequency-independent matrices. In the case when all chemical potentials $\mu_{i}$ 's are different, $\mathbf{G}_{d}{ }^{i}$ and $\mathbf{G}_{c}$ can be determined by substituting Eq. (7) directly into Dyson equation (6a). 


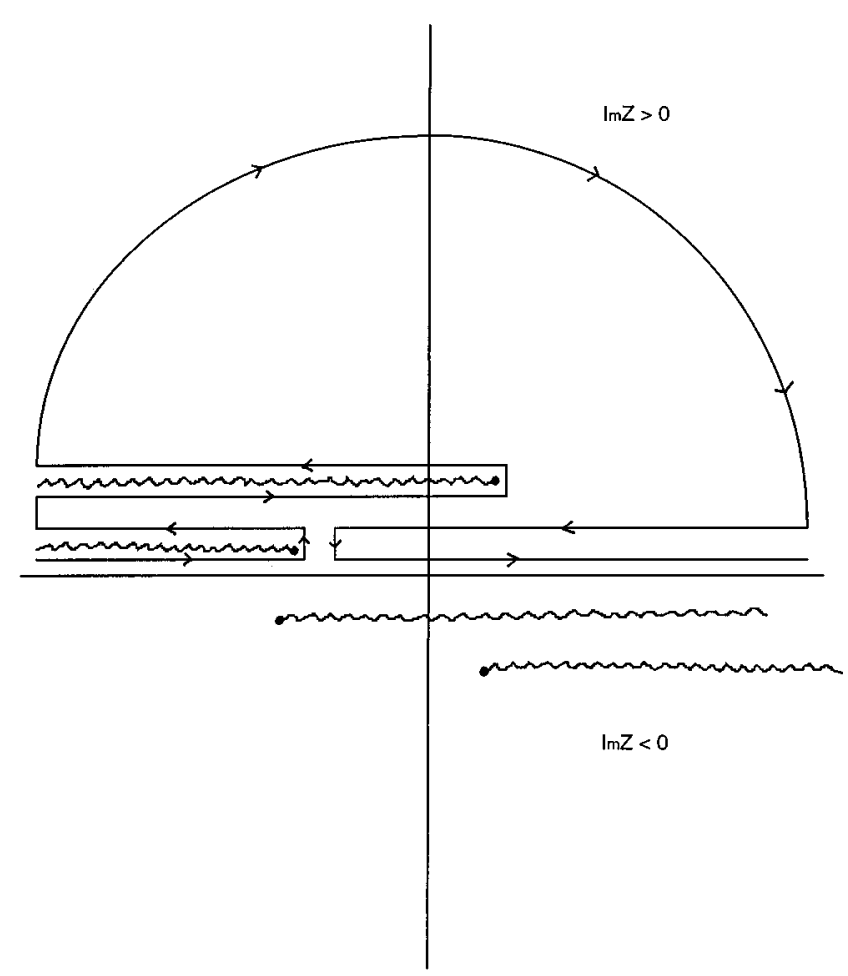

FIG. 1. The contour of integration to evaluate self-energy for a system with two different chemical potentials and four branch cuts.

Next we consider the closed-loop contribution $C\left(t_{f}-t_{i}\right)$. In the limit $t_{f}-t_{i} \rightarrow \infty, C(t) \rightarrow-i \Sigma t$, where $-i \Sigma$ represents the contribution of all closed loops, with no restriction on time except that one vertex has not been integrated over. ${ }^{12}$ According to usual perturbation theory, $\Sigma$ is the self-energy correction to the many-body wave function $\left|\psi_{o}\right\rangle$ coming from the interaction term $V_{\eta \eta^{\prime}}$. With Eq. (4), we obtain

$$
\frac{\partial \Sigma}{\partial \lambda}=-\int_{-\infty}^{\infty} \frac{d \omega}{2 \pi} \operatorname{Tr}\left(\mathbf{V G}^{\boldsymbol{\lambda}}(\omega)\right) e^{-i \omega 0^{-}},
$$

which is Hellmann-Feyman theorem. Integrating over $d \lambda$, we obtain

$$
\Sigma=-i \int_{-\infty}^{\infty} \frac{d \omega}{2 \pi} \operatorname{Tr} \ln (\mathbf{I}-i \mathbf{g}(\omega) \mathbf{V}) e^{-i \omega 0^{-}}
$$

The $d \omega$ integral can be further simplified by using the integration contour of Fig. 1. Details of the calculation can be find in Appendix A. For a system with $M(<N)$ different chemical potentials (recall that some of the Fermi seas can have the same chemical potential), we obtain

$$
\Sigma=-i \sum_{j=1}^{M} \int_{-\infty}^{\mu_{j}} \frac{d \boldsymbol{\epsilon}}{2 \pi} \operatorname{Tr} \ln \left\{\left[\mathbf{I}-i \mathbf{g}\left(\mu_{j}^{-}\right) \mathbf{V}\right]\left[\mathbf{I}-i \mathbf{g}\left(\mu_{j}^{+}\right) \mathbf{V}\right]^{-1}\right\} .
$$

The expression can be further simplified by separating out $\mathbf{g}\left(\mu_{j}^{ \pm}\right)$into two parts, $\mathbf{g}\left(\mu_{j}^{ \pm}\right)=\mathbf{g}_{c}\left(\mu_{j}\right) \pm \mathbf{g}_{d}\left(\mu_{j}\right)$, where $\mathbf{g}_{c}(\omega)$ is the part which remains unchanged as $\omega$ changes from $\mu_{j}^{-}$to $\mu_{j}^{+}$whereas $\mathbf{g}_{d}(\omega)$ is the part which changes discontinously. Using Eq. (6b), it is easy to see that

$$
\left[\mathbf{g}_{d}\left(\mu_{j}\right)\right]_{\eta \eta^{\prime}}=\delta_{\eta \eta^{\prime}} \delta\left(\mu_{j}-\mu_{\eta}\right) \nu_{o \eta} \pi
$$

and $\mathbf{g}_{c}\left(\mu_{j}\right)=\mathbf{g}\left(\mu_{j}^{+}\right)-\mathbf{g}_{d}\left(\mu_{j}\right)$. In terms of $\mathbf{g}_{c}$ and $\mathbf{g}_{d}$, Eq. (8) can be rewritten as

$$
\Sigma=-i \sum_{j=1}^{M} \int_{-\infty}^{\mu_{j}} \frac{d \epsilon}{2 \pi} \operatorname{Tr} \ln \left\{\left[\mathbf{I}-i \mathbf{T}\left(\mu_{j}\right)\right]\left[\mathbf{I}+i \mathbf{T}\left(\mu_{j}\right)\right]^{-1}\right\},
$$

where $\mathbf{T}\left(\mu_{j}\right)=\left[\mathbf{I}-i \mathbf{g}_{c}\left(\mu_{j}\right) \mathbf{V}\right]^{-1} \mathbf{g}_{d}\left(\mu_{j}\right) \mathbf{V}$. At equilibrium $\left(\mu_{\eta}=\mu\right.$ for all $\left.\eta\right), \mathbf{g}_{c}$ and $\mathbf{g}_{d}$ are just the imaginary and real parts of the Green's function matrix $\mathbf{g}(\omega)$ at $\omega=\mu^{+}$and the $\mathbf{T}(\mu)$ matrix thus defined is nothing but the usual $T$ matrix in scattering theory. The eigenvalues of the $T$ matrix are tan $\delta_{i}$ 's where $\delta_{i}$ 's are scattering phase shifts. In this case, we obtain

$$
\Sigma=-\sum_{i=1}^{N} \int_{-\infty}^{\mu_{i}} d \epsilon\left(\frac{\delta_{i}}{\pi}\right)
$$

which is just Fumi's theorem. ${ }^{11}$ The phase shifts are frequency independent because of our crude approximation for $g_{\eta}(t)$. The $\mathbf{T}\left(\mu_{j}\right)$ matrices we define here can be considered as generalization of the usual $T$ matrix to the nonequilibrium systems. Using our expression for $\mathbf{g}_{d}\left(\mu_{j}\right)$, it can be shown easily that the matrices $\mathbf{T}\left(\mu_{j}\right)$ 's are rank $n_{j}$ matrices, where $n_{j}$ is the number of Fermi seas with chemical potentials equal to $\mu_{j}$. Thus there are at most $n_{j}$ nonzero eigenvalues associated with each matrix $\mathbf{T}\left(\mu_{j}\right)$. In particular,

$$
\operatorname{Tr} \ln \left\{\left[\mathbf{I}-i \mathbf{T}\left(\mu_{j}\right) \mathbf{I}+i \mathbf{T}\left(\mu_{j}\right)\right]^{-1}\right\}=\sum_{i=1}^{n_{j}} \ln \frac{\left(1-i \tan \delta_{i}\right)}{\left(1+i \tan \delta_{i}\right)},
$$

where we have denoted the eigenvalues of $\mathbf{T}\left(\mu_{j}\right)$ by $\tan \delta_{i}$ 's as in the equilibrium case where $\delta_{i}$ 's are the corresponding phase shifts generalized to nonequilibrium systems. Notice that unlike usual (equilibrium) $T$ matrices, matrices $\mathbf{T}\left(\mu_{j}\right)$ 's are, in general, complex implying that the eigenvalues $\tan \delta_{j}$ 's and the corresponding phase shifts $\delta_{j}$ 's are also complex in general. The self-energy $\Sigma$ can be written in terms of the generalized phase shifts using Eq. (10) but is now a complex number because of the appearance of complex phase shifts, indicating that the nonequilibrium system we considered here has a finite lifetime, in agreement with general expectation that nonequilibrium systems are unstable and will relax to equilibrium states unless maintained by external forces. Notice also that the self-energy $\Sigma$ given by Eq. (10) is infinite because of divergence of the $d \epsilon$ integral as $\epsilon \rightarrow-\infty$. This is originated from our crude approximation for $g_{\eta}(t)$, which is supposed to be valid only in the long time or low frequency $(\epsilon \rightarrow 0)$ limit. The divergence can be regularized by introducing a mean self-energy $\Sigma_{m}$, with

$$
\Sigma_{m}=-\sum_{i=1}^{N} \int_{-\infty}^{\mu_{m}} d \epsilon\left(\frac{\delta_{i}}{\pi}\right),
$$

where $\mu_{m}=\sum_{i=1}^{N} \mu_{i} / N$ is the mean-chemical potential and

$$
\Sigma=\Sigma_{m}-\sum_{i=1}^{N} \frac{\delta_{i}}{\pi} \times\left(\mu_{i}-\mu_{m}\right) .
$$


$\Sigma_{m}$ can be calculated from Eq. (8) with $\mu_{j}$ replaced by $\mu_{m}$ and is in fact the self-energy for the system in equilibrium with all chemical potentials set equal to $\mu_{m}$, i.e., although $\delta_{i}$ 's are, in general, functions of $\mu_{i}$ 's, $\Sigma_{i} \delta_{i}=$ real constant, independent of whether the system is in a equilibrium or nonequilibrium state. To prove this identity we order the chemical potential $\mu_{j}$ 's in increasing order of magnitude $\left(\mu_{1}<\mu_{2}<\ldots<\mu_{M}\right)$. In this order $\mathbf{g}\left(\mu_{j}^{+}\right)=\mathbf{g}\left(\mu_{j+1}^{-}\right)$and it is easy to see that

$$
\begin{aligned}
\sum_{j=1}^{M} \operatorname{Tr} \ln \left\{\left[\mathbf{I}-i \mathbf{g}\left(\mu_{j}^{-}\right) \mathbf{V}\right]\left[\mathbf{I}-i \mathbf{g}\left(\mu_{j}^{+}\right) \mathbf{V}\right]^{-1}\right\} & \\
= & \left.\operatorname{Tr} \ln \left\{\mathbf{I}-i \mathbf{g}\left(\mu_{1}^{-}\right) \mathbf{V}\right]\left[\mathbf{I}-i \mathbf{g}\left(\mu_{M}^{+}\right) \mathbf{V}\right]^{-1}\right\}
\end{aligned}
$$

and the result follows. The divergence in $\Sigma$ implies that our approximate expression for $g_{\eta}(t)$ cannot be used to determine $\Sigma_{m}$ accurately, but can be used to determine the second, nonequilibrium contribution to the self-energy as long as the chemical potential differences between different Fermi seas are not too large.

To illustrate we consider a system of two reservoirs and study the $\mathbf{T}$ matrices and corresponding phase shifts in both equilibrium and out of equilibrium situations. To simplify the algebra we consider the special case with $\tan \theta_{1}$ $=\tan \theta_{2}=0$. With this simplification the $\mathbf{T}$ matrix in equilibrium is given by

$$
[\mathbf{T}]_{\eta \eta^{\prime}}=\nu_{o \eta} \pi V_{\eta \eta^{\prime}},
$$

with $\eta, \eta^{\prime}=1,2$ and the corresponding eigenvalues are

$$
\begin{aligned}
\tan \delta_{ \pm}= & \frac{1}{2}\left[\left(\nu_{o 1} \pi V_{11}+\nu_{o 2} \pi V_{22}\right) \pm\left[\left(\nu_{o 1} \pi V_{11}-\nu_{o 2} \pi V_{22}\right)^{2}\right.\right. \\
& \left.\left.+4 \nu_{01} \pi \nu_{o 2} \pi\left|V_{12}\right|^{2}\right]^{1 / 2}\right] .
\end{aligned}
$$

In the nonequilibrium case $\left(\mu_{1}>\mu_{2}\right)$ two rank one $\mathbf{T}$ matrices are present with

$$
\mathbf{T}\left(\mu_{1}\right)=\frac{1}{1-i \nu_{o 2} \pi V_{22}}\left(\begin{array}{cc}
\left(1-i \nu_{o 2} \pi V_{22}\right) \nu_{o 1} \pi V_{11} & \left(1-i \nu_{o 2} \pi V_{22}\right) \nu_{o 1} \pi V_{12} \\
i \nu_{o 1} \pi V_{11} \nu_{o 2} \pi V_{21} & i \nu_{o 1} \pi \nu_{o 2} \pi\left|V_{12}\right|^{2}
\end{array}\right)
$$

with one nonzero eigenvalue

$$
\tan \delta_{1}=\nu_{o 1} \pi V_{11}+i \frac{\nu_{o 1} \pi \nu_{o 2} \pi\left|V_{12}\right|^{2}}{1-i \nu_{o 2} \pi V_{22}}
$$

and

$$
\mathbf{T}\left(\mu_{2}\right)=\frac{1}{1+i \nu_{o 1} \pi V_{11}}\left(\begin{array}{cc}
-i \nu_{o 1} \pi \nu_{o 2} \pi\left|V_{12}\right|^{2} & -i \nu_{o 1} \pi V_{12} \nu_{o 2} \pi V_{22} \\
\left(1+i \nu_{o 1} \pi V_{11}\right) \nu_{o 2} \pi V_{21} & \left(1+i \nu_{o 1} \pi V_{11}\right) \nu_{o 2} \pi V_{22}
\end{array}\right)
$$

with the nonzero eigenvalue

$$
\tan \delta_{2}=\nu_{o 2} \pi V_{22}-i \frac{\nu_{o 1} \pi \nu_{o 2} \pi\left|V_{12}\right|^{2}}{1+i \nu_{o 1} \pi V_{11}}
$$

It is straightforward to show that $\delta_{+}+\delta_{-}=\delta_{1}+\delta_{2}$ and that the system has a finite lifetime proportional to $\left|\mu_{1}-\mu_{2}\right|$ as a result of having complex phase shifts. To understand the lifetime effect, we estimate the overlap between the initial wave function $\left|\psi_{o}\right\rangle$ and final state wave function $|\psi\rangle$ in the presence of the perturbation $V_{\eta \eta^{\prime}}$ in the above example. Before $V_{\eta \eta^{\prime}}$ is turned on, the ground state of our system consists of two Fermi seas 1 and 2, filled up to chemical potentials $\mu_{1}$ and $\mu_{2}$, respectively (we shall assume $\mu_{1}>\mu_{2}$ without loss of generality). Turning on the perturbation allows electrons with energy $\mu_{1}>E>\mu_{2}$ to tunnel from Fermi sea 1 to Fermi sea 2 , with tunneling probability $P \sim t^{+} t$, where $t$ is the transmission amplitude. Thus the overlap of the new ground state wave function with the original one will be reduced by a factor $\left(1-t^{+} t\right)^{N_{s}} \sim e^{-t^{+} t N_{s}}$, where
$N_{s}=$ total number of electrons with energy between $\mu_{1}$ and $\mu_{2} \sim\left(\mu_{1}-\mu_{2}\right) \times\left(N_{t} / D\right)$, where $N_{t}$ is the total number of electrons in the system and $D$ is the bandwidth. The exponential decay in overlap amplitude as a function of $\mu_{1}-\mu_{2}$ is consistent with the lifetime effect we observed in our theory. Notice that a similar lifetime effect has been discovered in the study of the nonequilibrium Kondo problem. ${ }^{2-4}$ In the present case, the lifetime effect appears as a natural consequence of having complex phase shifts in nonequilibrium $\mathbf{T}$ matrices.

\section{ASYMPTOTIC SOLUTION OF THE NONEQUILIBRIUM FERMI-EDGE SINGULARITY PROBLEM}

In this section we shall study the solution of the Dyson equation [Eq. (3)] for finite $t_{i}$ and $t_{f}$. Before going to the general solution let us first consider the case when the system is in equilibrium, i.e., all chemical potentials are equal. As in the infinite $t_{f}, t_{i}$ limit we first divide the unperturbed Green's function matrix $\mathbf{g}\left(t-t^{\prime}\right)$ into two parts, 


$$
\mathbf{g}\left(t-t^{\prime}\right)=\mathbf{g}_{c} \delta\left(t-t^{\prime}\right)+\mathbf{g}_{d}\left[\frac{-i}{\pi} P \frac{1}{t-t^{\prime}}\right],
$$

where $\mathbf{g}_{c}$ and $\mathbf{g}_{d}$ are the imaginary and real parts of the Green's function matrix $\mathbf{g}(\omega)$ at $\omega=0^{+}$. Notice that we have set the chemical potential at $\mu=0$ for simplicity. Substituting $\mathbf{g}$ into the Dyson equation and rearranging terms, we obtain

$$
\begin{aligned}
G_{\eta \eta^{\prime}}\left(t, t^{\prime}\right)= & \bar{g}_{\eta \eta^{\prime}}\left(t-t^{\prime}\right) \\
& +\sum_{\eta^{\prime \prime}} \frac{1}{\pi} T_{\eta \eta^{\prime \prime}} \int_{t_{i}}^{t_{f}} P \frac{1}{t-t^{\prime \prime}} G_{\eta^{\prime \prime} \eta^{\prime}}\left(t^{\prime \prime}, t^{\prime}\right) d t^{\prime \prime},
\end{aligned}
$$

where

$$
\bar{g}_{\eta \eta^{\prime}}\left(t-t^{\prime}\right)=\left[\left(\mathbf{I}-i \mathbf{g}_{c} \mathbf{V}\right)^{-1} \mathbf{g}\left(t-t^{\prime}\right)\right]_{\eta \eta^{\prime}}
$$

and

$$
T_{\eta \eta^{\prime}}=\left[\left(\mathbf{I}-i \mathbf{g}_{c} \mathbf{V}\right)^{-1} \mathbf{g}_{d} \mathbf{V}\right]_{\eta \eta^{\prime}}
$$

is the (equilibrium) $T$ matrix. Let $\mathbf{U}$ be the unitary tranformation which diagonalize the $T$ matrix, i.e., $\mathbf{U}^{+} \mathbf{T U}=\mathbf{t}$, where $t_{i j}=\delta_{i j} \tan \left(\delta_{i}\right)$. Performing the unitary transformation on Eq. (12a), we obtain

$$
\begin{aligned}
{\left[\mathbf{U}^{+} \mathbf{G}\left(t, t^{\prime}\right) \mathbf{U}\right]_{\eta \eta^{\prime}}=} & {\left[\mathbf{U}^{+} \overline{\mathbf{g}}\left(t-t^{\prime}\right) \mathbf{U}\right]_{\eta \eta^{\prime}}+\frac{\tan \delta_{\eta}}{\pi} } \\
& \times \int_{t_{i}}^{t_{f}} P \frac{1}{t-t^{\prime \prime}}\left[\mathbf{U}^{+} \mathbf{G}\left(t^{\prime \prime}, t^{\prime}\right) \mathbf{U}\right]_{\eta \eta^{\prime}} d t^{\prime \prime}
\end{aligned}
$$

Notice that the multicomponent matrix equation now becomes a single integral equation for each component $\left[\mathbf{U}^{+} \mathbf{G}\left(t, t^{\prime}\right) \mathbf{U}\right]_{\eta \eta^{\prime}}$ and has precisely the same form as the Dyson equation for the single Fermi sea Fermi-edge singularity problem studied by ND. ${ }^{12}$ In particular, for "unperturbed"' Green's function $\mathbf{U}^{+} \overline{\mathbf{g}}\left(t-t^{\prime}\right) \mathbf{U}$ with the general form

$$
\left[\mathbf{U}^{+} \overline{\mathbf{g}}\left(t-t^{\prime}\right) \mathbf{U}\right]_{\eta \eta^{\prime}}=a \delta\left(t-t^{\prime}\right)+b P \frac{1}{t-t^{\prime}},
$$

where $a, b$ are arbitrary constants, it was shown by ND that the longtime asymptotic solution has the general form

$$
\begin{aligned}
{\left[\mathbf{U}^{+} \mathbf{G}\left(t, t^{\prime}\right) \mathbf{U}\right]_{\eta \eta^{\prime}}=} & {\left[\mathbf{U}^{+} \mathbf{G}^{\infty}\left(t-t^{\prime}\right) \mathbf{U}\right]_{\eta \eta^{\prime}} } \\
& \times\left(\frac{\left(t_{f}-t^{\prime}\right)\left(t-t_{i}\right)}{\left(t_{f}-t\right)\left(t^{\prime}-t_{i}\right)}\right)^{\frac{\delta_{\eta}}{\pi}},
\end{aligned}
$$

where $\mathbf{U}^{+} \mathbf{G}^{\infty}\left(t-t^{\prime}\right) \mathbf{U}$ is the solution of Eq. (13) in the limit $t_{i} \rightarrow-\infty$ and $t_{f} \rightarrow \infty$. Notice that the solution is complete once $\mathbf{G}^{\infty}\left(t-t^{\prime}\right)$ and the eigenvalues and eigenvectors of the $T$ matrix are obtained. Physically the solution can be understood by visualizing the Green's function matrix $\mathbf{G}\left(t, t^{\prime}\right)$ as a sum of $N$ components where each component corresponds to an "eigenvector' of the $T$ matrix. The Dyson equation is "diagonalized" in this representation where each component of the matrix evolves in time separately according to its $T$ matrix eigenvalue $\tan \delta_{i}$ and with corresponding Fermi-edge singularity behavior characterized by $\delta_{i}$.

Next we argue that the solution of the Dyson equation in the longtime limit still has a similar form as Eq. (14) in a nonequilibrium situation. Physically, Fermi-edge singularities are longtime asymptotic effects coming from Fermi surface discontinuities and affects only (in frequency space) the behavior of Green's functions around Fermi surfaces. For a system with more than one Fermi surface, it is reasonable to expect that the Fermi-edge effect around one given chemical potential will not be affected much by other Fermi surfaces with different chemical potentials because of the mismatch in energy. In the following we shall prove by direct substitution that the Dyson equation in the nonequilibrium case indeed has a longtime asymptotic solution of the same form as Eq. (14). The solution is perturbatively connected to the unperturbed Green's functions $g_{\eta}(t)$ 's when $V(t) \rightarrow 0$ and reduces to the equilibrium solution when all chemical potentials are equal.

We assume that $\mathbf{G}\left(t, t^{\prime}\right)$ has the form ${ }^{14}$

$$
\begin{aligned}
\mathbf{G}\left(t, t^{\prime}\right)= & \sum_{i=1}^{N} \mathbf{G}_{d}^{i}\left(\frac{-i}{\pi} P \frac{1}{t-t^{\prime}}\right)\left(\frac{\left(t_{f}-t^{\prime}\right)\left(t-t_{i}\right)}{\left(t_{f}-t\right)\left(t^{\prime}-t_{i}\right)}\right)^{\delta_{i} / \pi} \\
& \times e^{-i \mu_{i}\left(t-t^{\prime}\right)}+\mathbf{G}_{c}
\end{aligned}
$$

where $\mathbf{G}_{d}{ }^{i}$, s and $\mathbf{G}_{c}$ are $N \times N$ matrices. Together with $\delta_{i}$ 's, these variables are to be determined self-consistently from the Dyson equation. Notice that $\mathbf{G}_{d}{ }^{i}$, s and $\mathbf{G}_{c}$ are defined in a way such that in the limit $t_{f(i)} \rightarrow \pm \infty$, they reduce to the same $\mathbf{G}_{d}{ }^{i}$, s and $\mathbf{G}_{c}$ s defined in Eq. (7). The following integral is useful in solving the Dyson equation:

$$
\begin{aligned}
& \int_{t_{i}}^{t_{f}} d t^{\prime \prime} P \frac{1}{t-t^{\prime \prime}} e^{-i \mu_{i}\left(t-t^{\prime \prime}\right)} P \frac{1}{t^{\prime \prime}-t^{\prime}}\left(\frac{\left(t_{f}-t^{\prime}\right)\left(t^{\prime \prime}-t_{i}\right)}{\left(t_{f}-t^{\prime \prime}\right)\left(t^{\prime}-t_{i}\right)}\right)^{\delta_{j} / \pi} e^{-i \mu_{j}\left(t^{\prime \prime}-t^{\prime}\right)}=i \pi t\left(\mu_{i}, \mu_{j}\right) P \frac{1}{t-t^{\prime}}\left[e^{-i \mu_{i}\left(t-t^{\prime}\right)}-e^{-i \mu_{j}\left(t-t^{\prime}\right)}\right. \\
& \left.\times\left(\frac{\left(t_{f}-t^{\prime}\right)\left(t-t_{i}\right)}{\left(t_{f}-t\right)\left(t^{\prime}-t_{i}\right)}\right) \delta^{\delta_{j} / \pi}\right]-\pi^{2} \delta\left(t-t^{\prime}\right)
\end{aligned}
$$


where

$$
t\left(\mu_{i}, \mu_{j}\right)= \begin{cases}\frac{i}{\tan \delta_{j}} & \text { for } \mu_{i}=\mu_{j}, \\ \operatorname{sgn}\left(\mu_{\imath}-\mu_{j}\right) & \text { for } \mu_{i} \neq \mu_{j} .\end{cases}
$$

For $\mu_{i} \neq \mu_{j}$, the integral is valid in the longtime limit when $\left(t_{f}-t\right),\left(t_{f}-t^{\prime}\right),\left(t-t_{i}\right)$ and $\left(t^{\prime}-t_{i}\right)$ are all large compared with $\left|\mu_{i}-\mu_{j}\right|^{-1}$. The details in evaluating this integral are given in Appendix B. Using this result, we find that Eq. (15) is indeed an asymptotic solution of the Dyson equation and the matrices $\mathbf{G}_{d}{ }^{i}, \mathbf{G}_{c}$ and phase shifts $\delta_{i}$ 's are determined by the following set of self-consistent equations:

$$
\begin{aligned}
{\left[\mathbf{I}-i \mathbf{g}_{i}\left(\mu_{i}\right) \mathbf{V}\right] \mathbf{G}_{d}^{(s)}\left(\mu_{i}\right)=} & \mathbf{g}_{d}\left(\mu_{i}\right)\left(\mathbf{I}+i \mathbf{V} \mathbf{G}_{c}\right) \\
& -i \sum_{j \neq i} \mathbf{g}_{r}\left(\mu_{j}\right) \mathbf{V} \mathbf{G}_{d}^{(s)}\left(\mu_{j}\right), \\
& {\left[\mathbf{I}-i \mathbf{g}_{i}(\infty) \mathbf{V}\right] \mathbf{G}_{c}=\mathbf{g}_{i}(\infty)+i \mathbf{g}_{r}(\infty) \mathbf{V} \sum_{j} \mathbf{G}_{d}^{(s)}\left(\mu_{j}\right), }
\end{aligned}
$$

and

$$
\mathbf{T}\left(\mu_{i}\right) \mathbf{G}_{\mathbf{d}}{ }^{i}=\tan \delta_{i} \mathbf{G}_{d}{ }^{i}
$$

for $i=1$ to $M$ where $\mathbf{G}_{d}^{(s)}\left(\mu_{i}\right)=\sum_{j} \mathbf{G}_{d}{ }^{j} \delta_{\mu_{j} \mu_{i}}, \mathbf{g}_{i}(\omega)$ and $\mathbf{g}_{r}(\omega)$ are the imaginary and real parts of the Green's function matrix $\mathbf{g}(\omega), \mathbf{g}_{d}\left(\mu_{i}\right)$ and $\mathbf{T}\left(\mu_{i}\right)$ are defined in the last section and are the "discontinuous" part of $\mathbf{g}(\omega)$ and the nonequilibrium $\mathbf{T}$ matrix at chemical potential $\mu_{i}$, respectively. The first two equations determine the $t_{f(i)} \rightarrow \pm \infty$ limit of the Green's function and can also be obtained directly by substituting Eq. (7) into Eq. (6a). Notice that in this limit, only $\mathbf{G}_{d}{ }^{(s)}$ can be determined unambigously from the Dyson equation but not individual $\mathbf{G}_{d}^{i}$, s except in the case when all $\mu_{i}$ 's are different. Individual $\mathbf{G}_{d}{ }^{i}$ 's are fixed from Eq. (17c) which states that $\mathbf{G}_{d}{ }^{i}$, s are eigenvectors of the $\mathbf{T}\left(\mu_{i}\right)$ matrix with eigenvalue $\tan \delta_{i}$. The phase shifts $\delta_{i}$ 's determine the longtime asymptotic behaviours of the Green's function $\mathbf{G}\left(t, t^{\prime}\right)$, in agreement with our physical expectation. We can also introduce (nonequilibrium) unitary $\mathbf{U}\left(\mu_{i}\right)$ matrices as in the equilibrium case which diagonalize the $\mathbf{T}\left(\mu_{i}\right)$ matrices. With $\mathbf{U}\left(\mu_{i}\right)$ it can be seen easily that the solution has exactly the same form as the equilibrium solution, the only difference being the appearance of complex phase shifts when the system is out of equilibrium. Notice that the restriction on time intervals in evaluating integral (16) implies that our nonequilibrium Green's function solution (15) can only be valid in the same longtime limit. The physical origin of this restriction can be understood by noting that Eq. (15) represents a solution where the Fermi-edge singularities on Fermi seas with different chemical potentials evolve independently from each other except through the generation of complex phase shifts. For two Fermi seas with chemical potential difference $\Delta \mu$, the Fermi-edge singularities can be resolved from each other only in the limit $\Delta t>(\Delta \mu)^{-1}$ because of the uncertainty principle, leading to same time restriction in our nonequilibrium Green's function solution.
In the Fermi-edge problem, the quantity of interests is the Green's function $\mathbf{F}\left(t_{f}, t_{i}\right)=G\left(t_{f}, t_{i}\right) e^{C\left(t_{f}, t_{i}\right)}$ which gives the $\mathrm{X}$-ray emission (absorption) spectrum. ${ }^{12}$ Putting $t=t_{f}$ and $t=t_{i}$ in Eq. (15), we find that the Green's function $\mathbf{G}\left(t_{f}, t_{i}\right)$ is diverging. As in the self-energy calculation, this spurious divergence comes from our crude treatment of short time behavior of $g_{\eta}(t) .^{12}$ To regularize the divergence, we follow ND and introduce a short-time cutoff $\left(i \xi_{o}\right)^{-1}$ in the Green's function $\mathbf{G}\left(t, t^{\prime}\right)$, obtaining in the limit $t \rightarrow t_{f}, t^{\prime} \rightarrow t_{i}$

$$
\mathbf{G}\left(t_{f}, t_{i}\right) \sim \sum_{j=1}^{N} \mathbf{G}_{d}^{j} \frac{-i}{\pi\left(t_{f}-t_{i}\right)}\left[i \xi_{o}\left(t_{f}-t_{i}\right)\right]^{2 \delta_{j} / \pi_{e}} e^{-i \mu_{j}\left(t_{f}-t_{i}\right)}
$$

in the limit $\xi_{o}\left(t_{f}-t_{i}\right) \gg 1$.

Next we consider finite $t_{f}, t_{i}$ corrections to the closedloop contribution $C\left(t_{f}-t_{i}\right)$. Following procedures employed by $\mathrm{ND},{ }^{12}$ we obtain (see Appendix C)

$$
C(t)=-i \Sigma^{m} t+\sum_{j=1}^{N}\left[i \frac{\delta_{j}}{\pi}\left(\mu_{j}-\mu_{m}\right) t-\left(\frac{\delta_{j}}{\pi}\right)^{2} \ln \left|\xi_{o} t\right|\right] .
$$

which has the same form as in equilibrium systems except that $\mu_{j}$ are not all equal and $\delta_{j}$ 's are, in general, complex in the present case.

The appearance of complex $\delta_{j}$ in $C(t)$ implies that the usual orthogonality catastrophy effect observed in equilibrium systems which is reflected in the $\ln \left|\xi_{o} t\right|$ term in the above equation will be superseded by a lifetime effect in the nonequilibrium case, where

$$
e^{C(t)} \sim\left(\frac{1}{\left(\xi_{o} t\right)}\right)^{\alpha} e^{-\Gamma t} e^{-i E t}
$$

where $\alpha=\Sigma_{j}\left(\delta_{j} / \pi\right)^{2}, \Gamma=\Sigma_{j}\left(\delta_{j}^{(2)} / \pi\right)\left(\mu_{j}-\mu_{m}\right)$ and $E$ are the imaginary and real parts of the self-energy, respectively. With Eq. (18) and Eq. (19), we obtain at longtime $t=t_{f}-t_{i}$,

$$
\begin{aligned}
F_{\eta \eta^{\prime}}(t) \rightarrow & \frac{-i}{\pi t} e^{-\Gamma t} e^{-i E t} \sum_{j=1}^{N}\left[\mathbf{G}_{\mathbf{d}}^{j}\right]_{\eta \eta^{\prime}} \\
& \times\left[i \xi_{o} t\right]^{\gamma_{j}^{(1)}} e^{-i\left(\mu_{j} t-\gamma_{j}^{(2)} \ln \left|\xi_{o} t\right|\right)},
\end{aligned}
$$

where $\quad \gamma_{i}^{(1)}=2 \delta_{i}^{(1)} / \pi-\Sigma_{j}\left(\delta_{j}^{(1) 2}-\delta_{j}^{(2) 2}\right) / \pi^{2} \quad$ and $\quad \gamma_{i}^{(2)}$ $=2 \times\left(\delta_{i}^{(2)} / \pi-\Sigma_{j} \delta_{j}^{(1)} \delta_{j}^{(2)} / \pi^{2}\right)$. Equation (20) is the main result of our paper. It shows that Fermi-edge singularity in general systems with more than one chemical potential can be described by generalized phaseshifts which are real for equilibrium systems and become complex when the system is out of equilibrium. Physically, complex phase shifts originate from the fact that nonequilibirum systems have finite lifetime in the absence of external maintaining forces. The effects of complex phase shifts will be considered in more detail in the next section where some applications of our result will be discussed. 


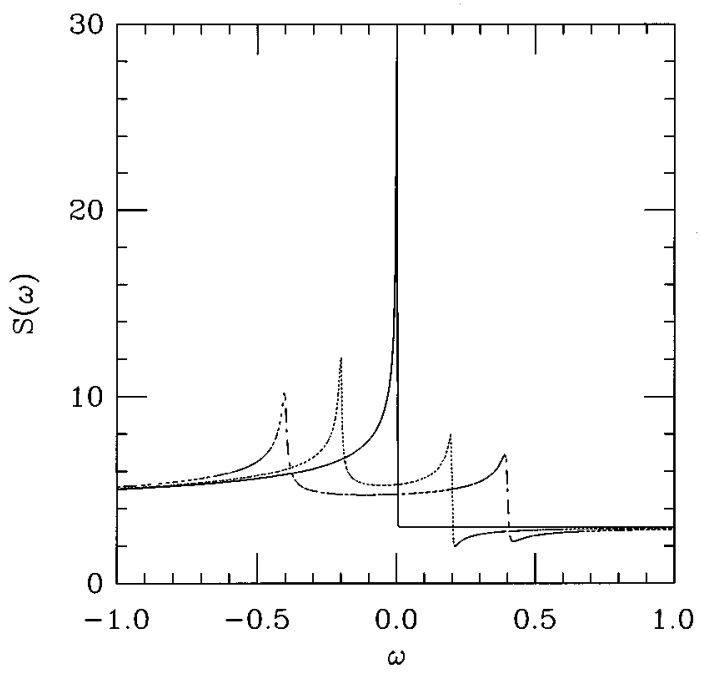

FIG. 2. Spectral function $S(\omega)$ for three different values of voltage differences: $\Delta \mu=0$ (equilibrium)(solid line), $\Delta \mu=0.4$ (shortdash line), and $\Delta \mu=0.8$ (long-dash line). We have set $\nu_{o 1} \pi V_{11}=\nu_{o 2} \pi V_{22}=1.0$ and $\nu_{01} \pi V_{12}=0.3$ in the calculation. The figure is drawn in arbitrary units.

\section{APPLICATIONS AND CONCLUSION}

Fermi-edge singularity can be observed in x-ray absorption or emission experiments where the x-ray emission spectrum of a deep localized state $S_{\eta \eta^{\prime}}(\omega)$ is given by

$$
S_{\eta \eta^{\prime}}(\omega)=\operatorname{Re} F_{\eta \eta^{\prime}}(-\omega),
$$

where $F_{\eta \eta^{\prime}}(\omega)$ is the Fourier transform of the Green's function $\mathbf{F}(t) \theta(t) .{ }^{12}$ The corresponding absorption spectrum can be obtained by replacing $\mathbf{G}_{d}{ }^{i}$ by $\mathbf{g}_{d}{ }^{i}$ and $\Gamma$ by $-\Gamma$ in Eq. (20) and Fourier transforming the corresponding function $\mathbf{F}^{\prime}(t) \theta(-t) .{ }^{12}$ It is difficult to probe the subtle difference arised under nonequilibrium environment in mesoscopic systems using x-ray spectroscopy, because of limitation of energy resolution. However, the difference can be probed by a photoluminescence experiment where much finer resolution can be achieved. Fermi-edge singularity in equilibrium mesoscopic systems has been observed in, for example, photoluminescence experiments on excitonic recombination of localized photoexcited holes in a single quantum well structure. ${ }^{17}$ A corresponding nonequilibrium experiment can be achieved by performing the same experiment on a double quantum well structure, so that two Fermi seas are involved in the photoluminescence process and voltage difference between the two Fermi seas can be set up by applying voltage bias.

Fourier transforming $\mathbf{F}(t) \theta(t)$, we obtain

$$
F_{\eta \eta^{\prime}}(\omega)=-\sum_{j=1}^{N} \frac{-i}{\pi}\left[\mathbf{G}_{\mathbf{d}}^{j}\right]_{\eta \eta^{\prime}} e^{i \pi \gamma_{j} \Gamma\left(\gamma_{j}\right)}\left(\frac{\xi_{o}}{\omega-\mu_{j}+i \Gamma}\right)^{\gamma_{j}}
$$

where $\gamma_{j}=\gamma_{j}^{(1)}+i \gamma_{j}^{(2)}$ and $\Gamma(z)$ is the usual gamma function analytic continued onto the complex z plane. We have set the energy $E=0$ in Eq. (21), i.e., the spectrum is measured with respect to $E$. The spectral function $S_{\eta \eta^{\prime}}(\omega)$ can be obtained from Eq. (21) in a straightforward way. Qualitatively, we find that the Fermi-edge singularity will be observed at all

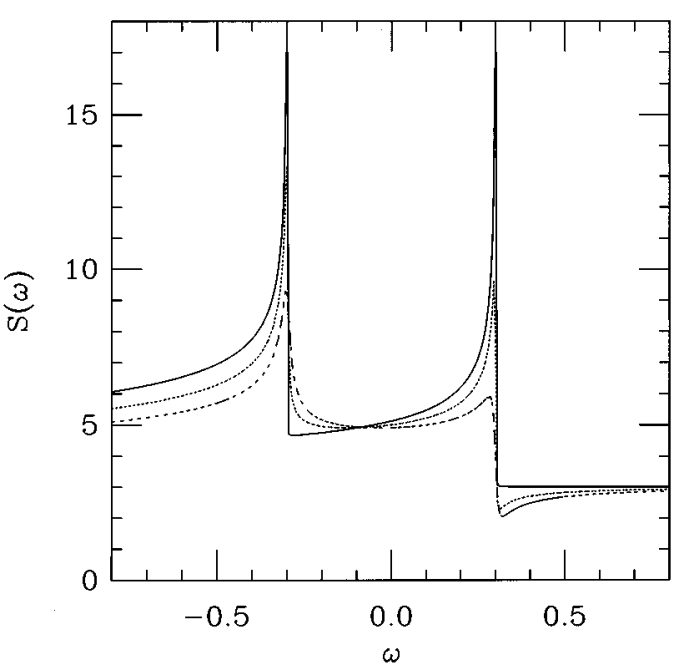

FIG. 3. Spectral function $S(\omega)$ for fixed voltage difference $\Delta \mu=0.6$ with three different values of $\nu_{o 1} \pi V_{12}: \nu_{o 1} \pi V_{12}=0$ (solid line), $\nu_{o 1} \pi V_{12}=0.2$ (short-dash line), and $\nu_{o 1} \pi V_{12}=0.4$ (long-dash line). We have set $\nu_{o 1} \pi V_{11}=\nu_{o 2} \pi V_{22}=1.0$ in the calculation. The figure is drawn in arbitrary units.

$N$ Fermi surfaces for a system out of equilibrium. However, the edge singularities are all broadened (asymmetrically) because of finite lifetime effect and the shape of the spectral functions are modified because of the interference effect associated with complex phase shifts. The broadening width is proportional to the electron tunneling probability between different Fermi seas, and to the voltage differences. To illustrate we consider a $N=2$ system and compute the total spectrum function

$$
S(\omega)=\sum_{\eta \eta^{\prime}} S_{\eta \eta^{\prime}}(\omega)
$$

in both equilibrium and nonequilibrium situations and the results are shown in Fig. 2 where the spectral function is plotted for different voltage differences $\Delta \mu$ 's. We have taken the approximation $g_{1}(t)=g_{2}(t)$ with $\tan \theta_{1(2)}=0$ and $V_{11}=V_{22}$. In Fig. 3 we show also the spectral function $S(\omega)$ at a fixed voltage difference $\Delta \mu$ as a function of the tunneling matrix element $V_{12}=V_{21}$. Notice that for $V_{12}=0$ the system consists of two separate (equilibrium) Fermi seas with different chemical potentials and $S(\omega)$ represents sum of the two (equilibrium) Fermi-edge singularities. The broadening of the Fermi-edge singularities and modification of the spectral function lineshape as a function of $\Delta \mu$ and $V_{12}$ is clear from two figures. Notice that our Fermi-edge singularity solution for $\Delta \mu \neq 0$ is exact only in the limit $\Delta \mu\left(t_{f}-t_{i}\right) \gg 1$. In the other limit $\Delta \mu\left(t_{f}-t_{i}\right) \ll 1$, the interference effect associated with the voltage difference between Fermi seas is neglectable and the solution of the Dyson equation should approach the equilibrium solution. Correspondingly, we expect that the spectral function $S(\omega)$ we computed for nonequilibrium systems is valid only in the frequency range $\left|\omega-\mu_{1(2)}\right|<\Delta \mu$. In the other limit $\left|\omega-\mu_{1(2)}\right| \gg \Delta \mu$, the correct spectral function is given by the "equilibrium" solution. In particular, in the limit when $\Delta \mu$ is small, the nonequilibrium spectral function (21) is valid over only a very limited range of frequencies. However 
it is obvious from Fig. 2 that the difference between equilibrium and nonequilibrium spectral functions is small at frequencies far away from the Fermi sea. Thus our nonequilibrium solution seems to represent the solution rather accurately at all frequencies.

In equilibrium condensed matter physics there exists a lot of phenomena which are closely related to the physics of Fermi-edge singularity or orthogonality catastrophe. ${ }^{18}$ For example, the problem of a two level system coupled to reservoir of electrons ${ }^{19}$ (dissipative tunneling problem) and Kondo effect. One may ask what will happen to these effects if the reservoir of electrons is in a nonequilibrium state ${ }^{2-4}$ as described in the present paper. It is obvious that the results obtained in this paper are relevant to these problems. For example, in the case of the $(N=2)$ nonequilibrium Kondo problem, $1 / N$ calculations suggest that there exists two Kondo peaks with the position pinned at the two Fermi surfaces. The Kondo peaks are broadened by the lifetime effect proportional to $\Delta \mu^{-1}$ which is very similar to the nonequilibrium lifetime effect discussed here. ${ }^{3,4}$ Our analysis suggests that these results are natural consequences of Fermiedge singularity in a nonequilibrium environment. A detailed study of the relation between nonequilibirum Fermi-edge singularity and the Kondo problem will be reported elsewhere.

\section{ACKNOWLEDGMENTS}

This work was supported by UPGC Hong Kong, through Grant No.UST636/94p.

\section{APPENDIX A: DERIVATION OF EQ. (8)}

We start with the expression for self-energy $\Sigma$,

$$
\Sigma=i \int_{-\infty}^{\infty} \frac{d \omega}{2 \pi} \operatorname{Tr} \ln (\mathbf{I}-i \mathbf{g}(\omega) \mathbf{V}) e^{-i \omega 0^{-}} .
$$

To evaluate the integral, we first consider the analytic properties of the integrand, or the Green's functions $g_{\eta}(\omega)$. $g_{\eta}(\omega)$ can be written in the spectral representation as

$$
g_{\eta}(\omega)=i \int_{\mu_{\eta}}^{\infty} d \epsilon \frac{\rho_{\eta}(\epsilon)}{(\omega-\epsilon+i \delta)}+i \int_{-\infty}^{\mu_{\eta}} d \epsilon \frac{\rho_{\eta}(\epsilon)}{(\omega-\epsilon-i \delta)},
$$

where $\rho_{\eta}(\epsilon)$ is the density of states at energy $\epsilon$ associated with Fermi sea $\eta$. On the complex plane, $g_{\eta}(z)$ has two branch cuts, one runs from $z=\mu_{\eta}-i \delta$ to $\infty-i \delta$, and is associated with the first term in Eq. (22). The other branch cut runs from $-\infty+i \delta$ to $\mu_{\eta}+i \delta$, and is associated with the second term. It is convenient to introduce for each function $g_{\eta}(\omega)$ a different $\delta_{\eta}$, so that the branch cuts from different Green's functions $g_{\eta}$ do not overlap with each other. The $\delta_{\eta}$ 's will all go to zero at the end of the calculation. In Fig. 1 we show an example of two Fermi seas where four branch cuts are found in the integrand associated with the two $g_{\eta}$ 's. The integral can be evaluated using the contour in Fig. 1 , where all the contributions cancel except those coming from differences between the top and bottom of branch cuts. For a system with $N$ Fermi seas the consideration is similar. The contribution to the integral is equal to the sum of the contributions from differences between the top and bottom of each branch cut which gives rise to Eq.(8).

\section{APPENDIX B: EVALUATION OF INTEGRAL (18)}

We want to evaluate in this appendix the integral

$$
\begin{aligned}
I= & \int_{t_{i}}^{t_{f}} d t^{\prime \prime} P \frac{1}{t-t \prime \prime} e^{-i \mu_{i}\left(t-t^{\prime \prime}\right)} P \frac{1}{t^{\prime \prime}-t^{\prime}} \\
& \times\left(\frac{\left(t_{f}-t^{\prime}\right)\left(t^{\prime \prime}-t_{i}\right)}{\left(t_{f}-t^{\prime \prime}\right)\left(t^{\prime}-t_{i}\right)}\right)^{\delta_{j} / \pi} e^{-i \mu_{j}\left(t^{\prime \prime}-t^{\prime}\right) .}
\end{aligned}
$$

In the case $\mu_{i}=\mu_{j}$, the integral has been studied by ND in the equilibrium Fermi-edge problem with the result given by Eqs. (16). We shall not repeat their calculation here but shall consider the case $\mu_{i} \neq \mu_{j}$. Using the identity

$$
\begin{aligned}
P \frac{1}{t-t^{\prime \prime}} P \frac{1}{t^{\prime \prime}-t^{\prime}}= & P \frac{1}{t-t^{\prime}} \times\left[P \frac{1}{t^{\prime \prime}-t^{\prime}}+P \frac{1}{t-t^{\prime \prime}}\right] \\
& -\pi^{2} \delta\left(t-t^{\prime \prime}\right) \delta\left(t^{\prime \prime}-t^{\prime}\right)
\end{aligned}
$$

we obtain

$$
\begin{aligned}
I= & e^{-i \mu_{i} t} e^{i \mu_{j} t^{\prime}}\left(\frac{t_{f}-t^{\prime}}{t^{\prime}-t_{i}}\right)^{\delta_{j} / \pi} P \frac{1}{t-t^{\prime}} \int_{t_{i}}^{t_{f}} d t^{\prime \prime} e^{-i\left(\mu_{j}-\mu_{i}\right) t^{\prime \prime}} \\
& \times\left(P \frac{1}{t^{\prime \prime}-t^{\prime}}+P \frac{1}{t-t^{\prime \prime}}\right)\left(\frac{t^{\prime \prime}-t_{i}}{t_{f}-t^{\prime \prime}}\right)^{\delta_{j} / \pi} \\
& -\pi^{2} \delta\left(t-t^{\prime}\right) .
\end{aligned}
$$

The integral $I$ involves integrals of the form

$$
I^{\prime}=\int_{t_{i}}^{t_{f}} d t^{\prime \prime} e^{i \Delta \mu t^{\prime \prime}}\left(\frac{t^{\prime \prime}-t_{i}}{t_{f}-t^{\prime \prime}}\right)^{\delta / \pi} P \frac{1}{t^{\prime \prime}-t}
$$

which cannot be evaluated exactly. However, the presence of the $e^{i \Delta \mu t^{\prime \prime}} P\left[1 /\left(t^{\prime \prime}-t\right)\right]$ term in the integrand suggests that contributions to the integral will be mostly coming from the region $\left|t^{\prime \prime}-t\right| \Delta \mu \leqslant 1$. Rewriting $I^{\prime}$ as

$$
I^{\prime}=\int_{t_{i}-t}^{t_{f}-t} d t^{\prime \prime} e^{i \Delta \mu\left(t^{\prime \prime}+t\right)}\left(\frac{t^{\prime \prime}+t-t_{i}}{t_{f}-t-t^{\prime \prime}}\right)^{\delta / \pi} P \frac{1}{t^{\prime \prime}},
$$

we find that in the limit $t_{f}-t, t-t_{i} \gg \Delta \mu^{-1}$, we can evaluate the integral by expanding the function

$$
\left(\frac{t^{\prime \prime}+t-t_{i}}{t_{f}-t-t^{\prime \prime}}\right)^{\delta / \pi}
$$

in a power series of $t^{\prime \prime}$. In the limit $t_{f}-t, t-t_{i} \rightarrow \infty$, we obtain

$$
I^{\prime} \rightarrow e^{i \Delta \mu t}\left(\frac{t-t_{i}}{t_{f}-t}\right)^{\delta / \pi} \int_{-\infty}^{\infty} d t^{\prime \prime} e^{i \Delta \mu t^{\prime \prime}} P \frac{1}{t^{\prime \prime}}
$$

where higher order contributions from expansion of $t^{\prime \prime}$ give rise to nonsingular corrections in ascending powers of $1 /\left[\Delta \mu\left(t_{f}-t\right)\right]$ or $1 /\left[\Delta \mu\left(t-t_{i}\right)\right]$. Repeating the same procedure for the integral $I$, we find in the limit $t_{f}-t, t_{f}-t^{\prime}, t-t_{i}, t^{\prime}-t_{i}$ all much larger than $\left|\mu_{i}-\mu_{j}\right|^{-1}$, 


$$
\begin{aligned}
I \rightarrow i & \pi \operatorname{sgn}\left(\mu_{i}-\mu_{j}\right) P \frac{1}{t-t^{\prime}}\left[e^{-i \mu_{i}\left(t-t^{\prime}\right)}-e^{-i \mu_{j}\left(t-t^{\prime}\right)}\right. \\
& \left.\times\left(\frac{\left(t_{f}-t^{\prime}\right)\left(t-t_{i}\right)}{\left(t_{f}-t\right)\left(t^{\prime}-t_{i}\right)}\right)^{\frac{\delta_{j}}{\pi}}\right]-\pi^{2} \delta\left(t-t^{\prime}\right)
\end{aligned}
$$

which is the result obtained in Eq. (16) for $\mu_{i} \neq \mu_{j}$.

\section{APPENDIX C: EVALUATION OF $C(t)$}

We consider the Green's function $\mathbf{G}\left(t, t^{\prime}\right)$ in the limit $t-t^{\prime} \rightarrow 0$. In this limit, it is easy to show that

$$
\mathbf{G}\left(t, t^{\prime}\right) \rightarrow \mathbf{G}^{\infty}\left(t-t^{\prime}\right)-i \sum_{j=1}^{N} \mathbf{G}_{d}^{j} \frac{\delta_{j}}{\pi^{2}}\left[\frac{1}{t_{f}-t}+\frac{1}{t-t_{i}}\right],
$$

where $\mathbf{G}^{\infty}\left(t-t^{\prime}\right)$ is the solution in the $t_{f(i)} \rightarrow \pm \infty$ limit. Corresponding, the contribution to $C(t)$ can be divided into two parts, the first part coming from $\mathbf{G}^{\infty}$ gives rise to the selfenergy $\Sigma$ which we discussed in detail in Sec. II. The second part $C^{(2)}\left(t_{f}-t_{i}\right)$ is a finite $t_{f(i)}$ correction. Using Eq. (4) and performing the integration over $d t$, we obtain

$$
\lambda \frac{\partial C^{(2)}(t)}{\partial \lambda}=-\sum_{j=1}^{N} \operatorname{Tr}\left[(\lambda \mathbf{V}) \mathbf{G}_{d}{ }^{j}\right] \frac{2 \delta_{j}}{\pi^{2}} \ln \left|\xi_{o} t\right|,
$$

where we have again introduced a short-time cutoff $\xi_{o}^{-1}$ in evaluating the $d t$ integral. Equation (C2) can be further simplified by the following trick. Notice that the nonequilibrium contribution to $C(t)$ from $\mathbf{G}^{\infty}$ is the self energy $\Sigma-\Sigma_{m}$ which can be expressed in terms of phase shifts by the (generalized) Fumi theorem, i.e.,

$$
\begin{aligned}
\lambda \frac{\partial\left(\Sigma-\Sigma_{m}\right)}{\partial \lambda}= & -\sum_{i=1}^{N} \operatorname{Tr}\left[(\lambda \mathbf{V}) \mathbf{G}_{\mathbf{d}}{ }^{i}\right] \frac{1}{\pi} \times\left(\mu_{i}-\mu_{m}\right)= \\
& -\sum_{i=1}^{N} \lambda \frac{\partial \delta_{i}}{\partial \lambda} \times \frac{1}{\pi}\left(\mu_{i}-\mu_{m}\right)
\end{aligned}
$$

Comparing this equation with Eq. (C2a), we obtain

$$
\frac{\partial C^{(2)}(t)}{\partial \lambda}=-\sum_{i=1}^{N}\left(\frac{\partial \delta_{i}}{\partial \lambda}\right)\left(2 \frac{\delta_{i}}{\pi^{2}}\right) \ln \left|\xi_{o} t\right|
$$

The integration over $d \lambda$ can now be carried out easily. Together with the contribution from $\mathbf{G}^{\infty}$, we obtain

$$
C(t)=-i \Sigma^{m} t+\sum_{j=1}^{N}\left[i \frac{\delta_{j}}{\pi}\left(\mu_{j}-\mu_{m}\right) t-\left(\frac{\delta_{j}}{\pi}\right)^{2} \ln \left|\xi_{o} t\right|\right] .
$$

which is Eq. (19).
${ }^{1}$ T.K. Ng, Phys. Rev. Lett. 68, 1021 (1992).

${ }^{2}$ S. Hershfield, J.H. Davies, and J.W. Wilkins, Phys. Rev. Lett. 67, 3720 (1991).

${ }^{3}$ Y. Meir, N.S. Wingreen, and P.A. Lee, Phys. Rev. Lett. 70, 2601 (1993).

${ }^{4}$ T.K. Ng, Phys. Rev. Lett. 70, 3635 (1993).

${ }^{5}$ D.C. Ralph and R.A. Buhrman, Phys. Rev. Lett. 74, 3401 (1994).

${ }^{6}$ C. de C. Chamon, D.E. Freed, and X.G. Wen, Phys. Rev. B 51, 2363 (1995).

${ }^{7}$ U. Hanke, Yu.M. Galperin, and K.A. Chao, Phys. Rev. B 50, 1995 (1994).

${ }^{8}$ N.S. Wingreen and Y. Meir, Phys. Rev. B 49, 11040 (1994).

${ }^{9}$ M. Büttiker, A. Pretre, and H. Thomas, Phys. Rev. Lett. 70, 4114 (1993); Phys. Lett. A 180, 364 (1993).
${ }^{10}$ A.P. Jauho, N.S. Wingreen, and Y. Meir, Phys. Rev. B 50, 5528 (1994).

${ }^{11}$ See, for example, G. D. Mahan, Many-Particle Physics (Plenum, New York, 1981).

${ }^{12}$ P. Noziéres and C. T. De Dominicis, Phys. Rev. 178, 1097 (1969).

${ }^{13}$ See, for example, K. Ohtaka and Y. Tanabe, Rev. Mod. Phys. 62, 929 (1990).

${ }^{14}$ T.K. Ng, Phys. Rev. B 51, 2009 (1995).

${ }^{15}$ C. Tanguy and M. Combescot, Phys. Rev. Lett. 68, 1935 (1992).

${ }^{16}$ J. P. Foing et al., Phys. Rev. Lett. 68, 110 (1992).

${ }^{17}$ P. A. Folkes et al., Phys. Rev. Lett. 71, 3379 (1993).

${ }^{18}$ P. W. Anderson, Phys. Rev. Lett. 18, 1049 (1967).

${ }^{19}$ A. J. Leggett et al., Rev. Mod. Phys. 59, 1 (1987). 